\title{
Oxidative metabolism drives inflammation-induced platinum resistance in human ovarian cancer
}

\author{
DS Matassa ${ }^{1,8}$, MR Amoroso ${ }^{1,8}, \mathrm{H} \mathrm{Lu}^{2}$, R Avolio ${ }^{1}, \mathrm{D}$ Arzeni ${ }^{1}, \mathrm{C}$ Procaccini ${ }^{3}$, D Faicchia ${ }^{4}, \mathrm{~F}$ Maddalena ${ }^{5}$, V Simeon ${ }^{5}$, I Agliarulo ${ }^{1}$, E Zanini ${ }^{2}$, \\ C Mazzoccoli ${ }^{5}$, C Recchi ${ }^{2}$, E Stronach ${ }^{6}$, G Marone $^{4}$, H Gabra $^{6}$, G Matarese ${ }^{1}$, M Landriscina ${ }^{*, 5,7}$ and F Esposito*,1
}

Tumour cells have long been considered defective in mitochondrial respiration and mostly dependent on glycolytic metabolism. However, this assumption is currently challenged by several lines of evidence in a growing number of tumours. Ovarian cancer (OC) is one of the most lethal cancers worldwide, but it continues to be a poorly understood disease and its metabolic features are far to be elucidated. In this context, we investigated the role of tumour necrosis factor receptor-associated protein 1 (TRAP1), which is found upregulated in several cancer types and is a key modulator of tumour cell metabolism. Surprisingly, we found that TRAP1 expression inversely correlated with grade, stage and lower survival in a large cohort of OC patients. Accordingly, TRAP1 silencing induced resistance to cisplatin, resistant cells showed increased oxidative metabolism compared with their sensitive counterpart, and the bioenergetics cellular index of higher grade tumours indicated increased mitochondrial respiration. Strikingly, cisplatin resistance was reversible upon pharmacological inhibition of mitochondrial oxidative phosphorylation by metformin/ oligomycin. At molecular level, increased oxidative metabolism in low TRAP1-expressing OC cells and tissues enhanced production of inflammatory mediators such as interleukin (IL)-6 and IL-8. Mechanistically, we identified members of the multidrug resistance complex (MDR) as key mediators of such metabolism-driven, inflammation-induced process. Indeed, treatment of OC cell lines with TNF $\alpha$ and IL6 induced a selective increase in the expression of TAP1 and multidrug resistance protein 1, whereas TAP1 silencing sensitized cells to cisplatin-induced apoptosis. Our results unveil a novel role for TRAP1 and oxidative metabolism in cancer progression and suggest the targeting of mitochondrial bioenergetics to increase cisplatin efficacy in human $\mathrm{OC}$. Cell Death and Differentiation (2016) 23, 1542-1554; doi:10.1038/cdd.2016.39; published online 20 May 2016

The current paradigmatic view, based on the classic observations of Otto Warburg, recognizes tumour cells as defective in mitochondrial respiration and mostly relying on glycolytic metabolism. However, very recent observations provide opposite evidence showing that melanoma cells are critically dependent on oxidative phosphorylation (OXPHOS) rather than glycolysis ${ }^{1,2}$ and that respiratory function is essential for tumorigenic and metastatic potential of breast cancer and melanoma cells. ${ }^{3,4}$ Notably, it has been shown that the most aggressive ovarian cancer (OC) cell lines show a marked dependence on glutamine rather than on glucose, ${ }^{5}$ and cancer stem cells from epithelial OC (EOC) patients privilege OXPHOS and resist glucose deprivation, ${ }^{6}$ suggesting that glycolysis is not the main pathway sustaining tumour growth in these systems. EOC is the fifth most common malignancy in women worldwide and, as the introduction of platinum-based chemotherapy, only modest increases in overall survival have been registered. ${ }^{7}$ Indeed, EOC is considered a chemo-responsive neoplasm, but, despite this, the majority of patients ultimately develop recurrent disease, with over $60 \%$ of women eventually dying from their disease within 5 years of diagnosis. ${ }^{7}$ In this context, the identification of reliable biomarkers that would allow to tailor treatment according to the patient's disease is urgently needed. Interestingly, high expression of the tumour necrosis factor receptor-associated protein 1 (TRAP1) has been often proposed as a biomarker predictive for poor outcome, among others, in colorectal, ${ }^{8}$ hepatocellular, ${ }^{9}$ non-small cell lung $^{10}$ and prostate ${ }^{11}$ carcinomas. Moreover, TRAP1 has been recognized as a positive regulator of expression ${ }^{12}$ and activity ${ }^{13}$ of the oncogene BRAF, as a factor favouring resistance to taxanes ${ }^{14}$ and anthracyclins ${ }^{15}$ in breast cancer

${ }^{1}$ Dipartimento di Medicina Molecolare e Biotecnologie Mediche, Università di Napoli 'Federico Il', Napoli, Italy; ${ }^{2}$ Imperial College London, Ovarian Cancer Action Research Centre, Department of Cancer and Surgery, Institute of Reproductive and Developmental Biology, London, UK; ${ }^{3}$ Laboratorio di Immunologia, Istituto di Endocrinologia e Oncologia Sperimentale, Consiglio Nazionale delle Ricerche (IEOS-CNR), Napoli, Italy; ${ }^{4}$ Dipartimento di Scienze Mediche Traslazionali, Centro Interdipartimentale di Ricerca in Scienze Immunologiche di Base Cliniche (CISI), Università di Napoli 'Federico II', Napoli, Italy; '5aboratorio di Ricerca Preclinica e Traslazionale, IRCCS-CROB, Centro di Riferimento Oncologico della Basilicata, Rionero in Vulture, PZ Italy; ${ }^{6}$ Ovarian Cancer Action Research Centre, Department of Surgery and Cancer, Imperial College London, London, UK and ${ }^{7}$ Dipartimento di Scienze Mediche e Chirurgiche, Università degli Studi di Foggia, Foggia, Italy

*Corresponding author: M Landriscina, Dipartimento di Scienze Mediche e Chirurgiche, Università degli Studi di Foggia, Viale Pinto, 1, Foggia 71100, Italy. Tel: +39881 736241; Fax: +39 881 733614. E-mail: matteo.landriscina@unifg.it

or F Esposito, Dipartimento di Medicina Molecolare e Biotecnologie Mediche, Università di Napoli Federico II, Via S Pansini 5, Napoli 80131, Italy. Tel: +39 81 7463145; Fax: +39 81 7464359; E-mail: franca.esposito@ unina.it

${ }^{8}$ These authors contributed equally to this work.

Abbreviations: OXPHOS, oxidative phosphorylation; HGSOC, high-grade serous ovarian cancer; TRAP1, tumour necrosis factor receptor-associated protein 1; OC, ovarian cancer; EOC, epithelial ovarian cancer; PFS, progression-free survival; TCGA, The Cancer Genome Atlas; BEC, Bioenergetic Cellular index; ECAR, extracellular acidification rate; OCR, oxygen consumption rate; MDR1, multidrug resistance protein 1; ROS, reactive oxygen species

Received 22.12.15; revised 07.3.16; accepted 21.3.16; Edited by E Gottlieb; published online 20.5.2016 
a

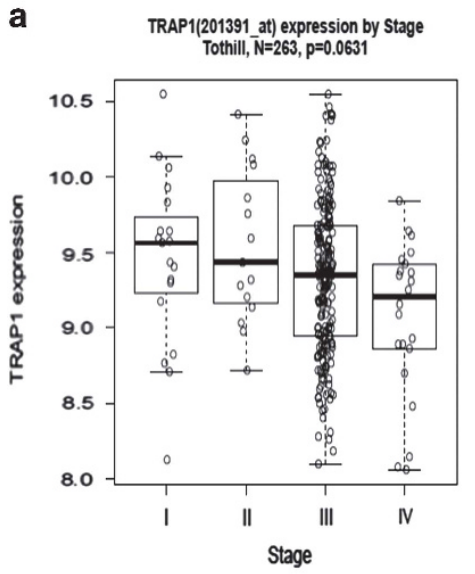

d

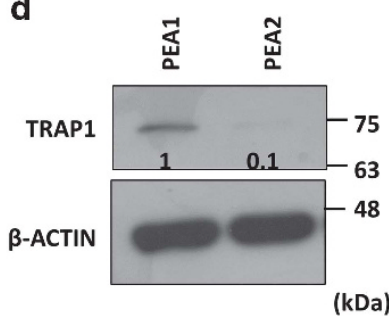

b
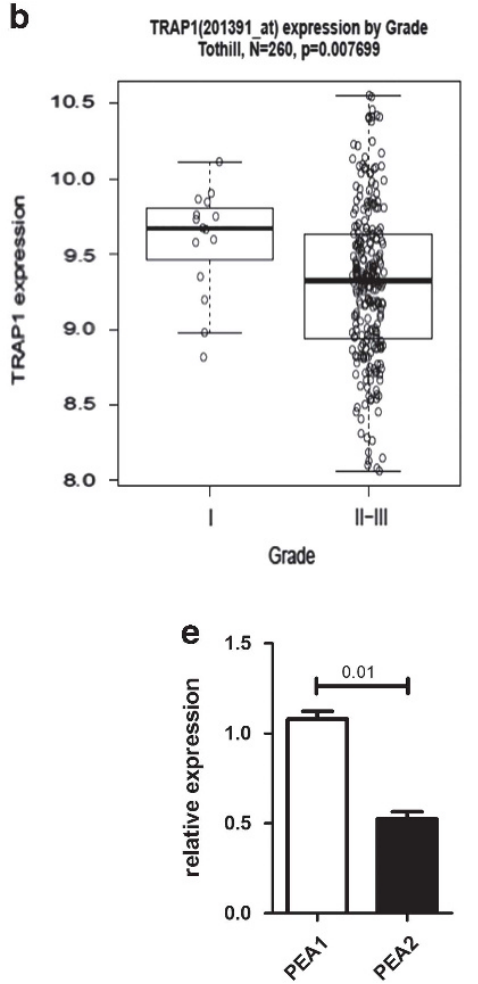

C
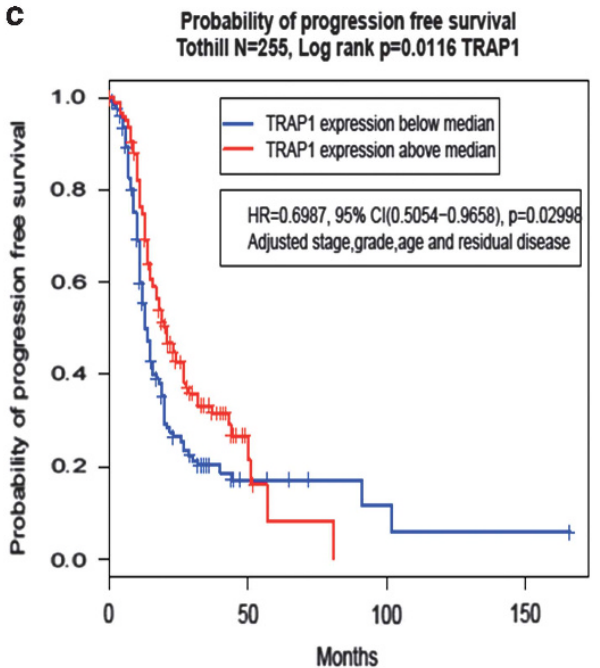

Figure 1 Lower TRAP1 expression is associated with more advanced disease. (a) Correlation between TRAP1 mRNA expression and tumour stage from the Tothill data set ( $P$-values determined by the Kruskal-Wallis test). (b) Correlation between TRAP1 mRNA expression and tumour grade from the Tothill data set ( $P$-values determined by the Wilcoxon rank sum test). (c) Kaplan-Meier estimates of the impact of TRAP1 on PFS ( $P$-values determined by the log-rank test). (d) Total lysates obtained from PEA1-sensitive and PEA2-resistant cells were separated by SDS-PAGE and immunoblotted with the indicated antibodies. Images are representative of three independent experiments. (e) Realtime RT-PCR analysis of TRAP1 mRNAs expression in PEA1-sensitive and PEA2-resistant cells. Data are expressed as mean \pm S.E.M. from three independent experiments with technical triplicates each. Numbers above bars indicate the statistical significance $(P$-value), based on the two-tailed Student's $t$-test

and a regulator of cell migration and tumour metastases. ${ }^{16,17}$ However, whether TRAP1 roles in tumour development and progression are generally oncogenic or not is still a matter of debate. Recently, it has been reported an inverse correlation between TRAP1 expression and tumour stage in cervical, bladder and clear cell renal cell carcinoma. ${ }^{18}$ Among them, remarkably, cervical carcinoma relies mostly on OXPHOS for its energetic metabolism. ${ }^{19}$ This is of particular interest, because TRAP1 is an important determinant of tumour metabolism ${ }^{20}$ and has been proposed as a pivotal driver of cancer cell's shift from OXPHOS toward aerobic glycolysis, the so-called Warburg effect. ${ }^{21}$ Interestingly, recent data also directly correlate TRAP1 expression and overall survival of OC patients. ${ }^{22}$ Altogether, these observations prompt us to raise the hypothesis that TRAP1-dependent regulation of cellular metabolism and its role in cancer development/ progression could be mutually related. Herein, we demonstrate that TRAP1 expression and the consequent metabolic rewiring control platinum resistance and tumour progression through an inflammation-mediated mechanism. By addressing several open questions in human OC, this study could provide relevant knowledge for novel therapeutic approaches based on the targeting of mitochondrial bioenergetics to improve drug efficacy in tumours with increased oxidative metabolism.

\section{Results}

TRAP1 expression associates with OC progression and survival. Preliminary analyses in a subset of OC tissues indicated that high TRAP1 expression has a positive impact on chemotherapy response and overall survival, ${ }^{22}$ oppositely to what well demonstrated in colorectal, prostate and many other cancers. ${ }^{23}$ To shed further light on TRAP1 functions in OC, we took advantage of publicly available serous ovarian cancer cases from Tothill dataset. We used gene expression microarray data (263 samples) to perform correlation analyses between TRAP1 expression and tumour stage, grade and survival. Surprisingly, data showed that lower TRAP1 expression is associated with more advanced disease in OC samples (Figure 1); more specifically, TRAP1 expression has an inverse tendency with tumour stage $(N=263, P=0.0631)$ (Figure 1a), a significant inverse correlation with grade $(N=260, P=0.007699)$ (Figure $1 b)$, and it is associated with better progression-free surviree survival (PFS) (Figure 1c) $(N=255, P=0.0116)$, this also suggesting that higher TRAP1 expression associates with better chemotherapy response. A significant separation of PFS curve before 50 months is clinically relevant, considering that the median PFS for high grade serous OC (HGSOC) patients is 15 months. These findings confirm an intriguing role for TRAP1 in OC progression and outcome. 
a

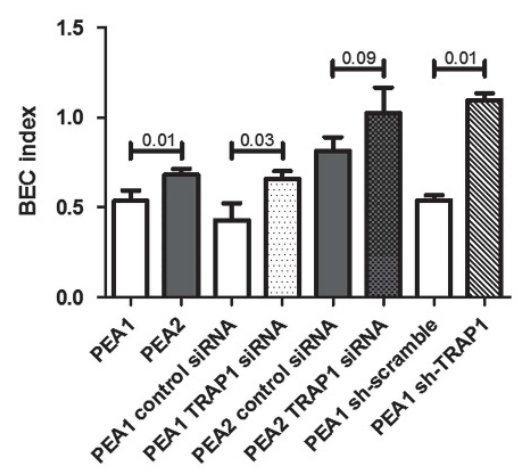

b

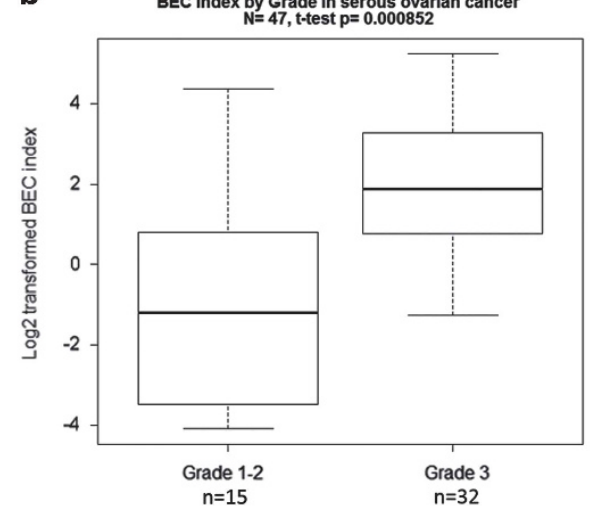

c

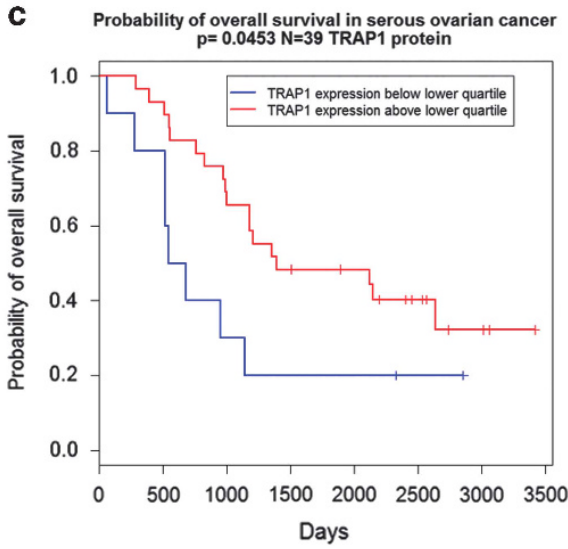

Figure 2 Drug resistance and tumour grade are associated with cell metabolism in OC. (a) BEC index analysis on PEA1 and PEA2 cells upon TRAP1 silencing. Where indicated, cells were transfected with TRAP1-directed or control siRNAs; $72 \mathrm{~h}$ after transfection, cells were harvested and total protein lysates were immunoblotted with anti$\beta$ F1ATPase, anti-HSP60 and anti-GAPDH antibodies. Immunoreactive bands were quantified by using ImageJ and BEC index was calculated by the formula F1ATPase/HSP60/ GAPDH (see Material and Methods section for details). Data are expressed as mean \pm S.E.M. from five independent experiments. Numbers above bars indicate the statistical significance ( $P$-value), based on the two-tailed Student's $t$-test. (b) Total lysates obtained by a set of OC tissues $(n=47)$ were analyzed by WB analysis for BEC index calculation as described in panel (a). The resulting BEC index values were normalized on a reference sample and correlated to clinical parameters of each sample. (c) Kaplan-Meier estimates of the impact of TRAP1 on Overall Survival ( $P$-values determined by the log-rank test)

To further characterize TRAP1 relevance in OC biology, we used as a model two OC matched cell lines obtained from the same patient with HGSOC before and after development of clinical platinum resistance (PEA1/PEA2). ${ }^{24}$ We analyzed TRAP1 expression levels in both cell lines and, in agreement with our patients' survival analysis, we found that platinumresistant OC PEA2 cells show lower TRAP1 expression levels than their sensitive counterpart PEA1, both at protein and mRNA levels (Figures 1d and e).

Low TRAP1 expression correlates with oxidative metabolism. Data from public gene expression databases display that TRAP1 expression inversely correlates with tumour stage in cervical cancer, ${ }^{18}$ that, interestingly, has been shown to prevalently rely on OXPHOS for its energy metabolism, ${ }^{19}$ whereas TRAP1 is an important driver of glycolytic metabolism. ${ }^{21}$ Therefore, we hypothesized that TRAP1 could act as a tumour suppressor in a context in which OXPHOS is the preferential pathway for ATP source. To this aim, metabolic features of OC cells were investigated by the BioEnergetic Cellular (BEC) index calculation, obtained through densitometric analysis of the ratio between F1ATPase, HSP60 and GAPDH expression levels: a higher $B E C$ index correlates with an increased dependence on respiratory capacity. The BEC index has been exploited as prognostic value in several cancer types, including OC. ${ }^{25} \mathrm{We}$ found that the BEC index is significantly increased upon siRNA-mediated TRAP1 silencing in PEA1 and also tend to increase in PEA2 cells, indicating a bioenergetic shift toward OXPHOS, and analogous results were obtained in shmediated stable TRAP1 silencing in PEA1 cells (Figure 2a). Moreover, the same analysis indicates for PEA2 cells a $B E C$ index value higher than their sensitive counterpart PEA1, which correlates with TRAP1 expression levels, thus suggesting a predominant oxidative metabolism in chemoresistant cells (Figure 2a) and supporting TRAP1 role in downregulation of mitochondrial activity.

To verify the relevance of TRAP1 and metabolism roles in patients, we analyzed $47 \mathrm{HGSOC}$ tissue specimens for TRAP1 expression and for BEC index markers by WB. Remarkably, a positive and statistically significant correlation was found between tumour grade and BEC index (Figure 2b), whereas high TRAP1 protein expression correlated with better overall survival in the same patients (Figure 2c). These data support the in vivo relevance of our findings in human pathology, and indicate that in more aggressive tumours TRAP1 expression decreases and BEC index increases, suggesting altogether a shift toward OXPHOS metabolism in advanced OC.

TRAP1 is responsible for a metabolic shift in OC cells. To further validate TRAP1 role in OC bioenergetics, we performed metabolic analyses in our paired cell lines. The extracellular acidification rate (ECAR), an indicator of glycolysis, and oxygen consumption rate (OCR) were measured using the Seahorse XFe96 Bioanalyzer (North Billerica, MA, USA). First, PEA1 and PEA2 bioenergetics was analyzed to understand whether low TRAP1 background in chemoresistant cells is correlated to a metabolic shift toward OXPHOS. ECAR analysis shows a higher glycolytic capacity (either basal or maximal) in PEA1 compared with PEA2 (Figure 3a); furthermore, OCR value is significantly higher in PEA2 cells compared with PEA1 cells (Figure $3 b$ ), thus indicating again that chemoresistant cells, with low TRAP1 levels, shifted more toward mitochondrial function for ATP production. To further support the impact of TRAP1 expression on OC cells' bioenergetics, the same Seahorse analysis was performed on PEA1 cells upon siRNA-mediated TRAP1 silencing. Results show that TRAP1 silencing does not affect glycolytic activity (Figure 3c), whereas both basal and maximum OCR are significantly increased (Figure $3 d$ ). These results confirm 
a

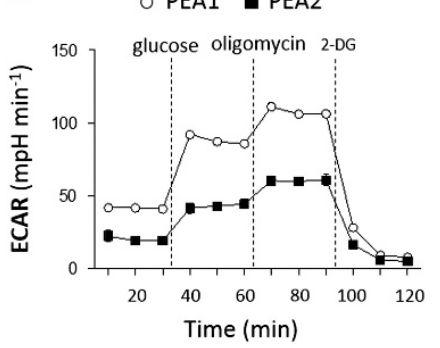

b

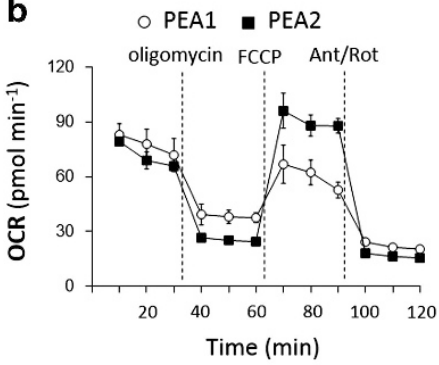

c $O$ control siRNA - TRAP1 siRNA

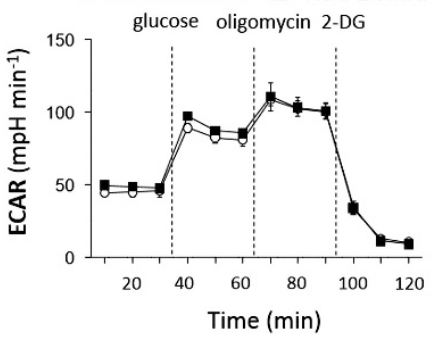

d o control siRNA - TRAP1 SIRNA oligomycin FCCP Ant/Rot

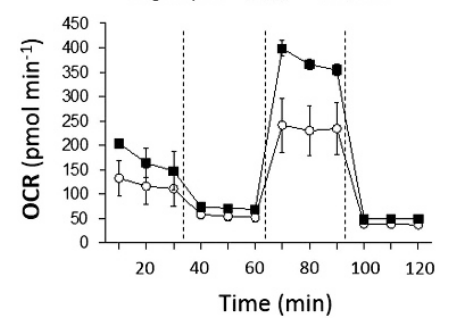

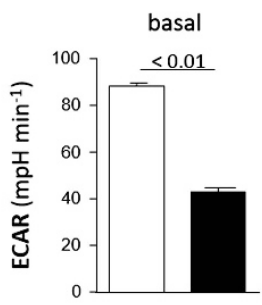
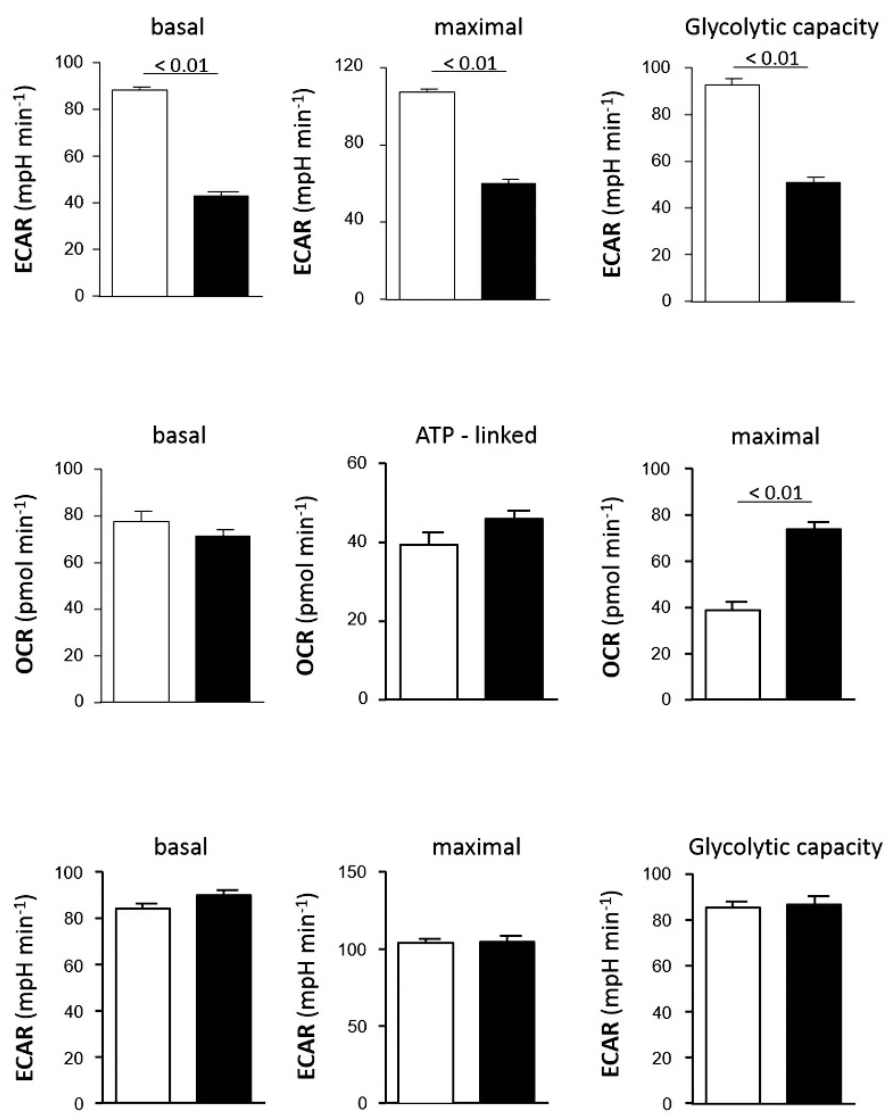

Figure 3 Modulation of TRAP1 expression causes metabolic shift in OC cells. (a and $\mathbf{b}$ ) Kinetic profile of ECAR and OCR in PEA1 and PEA2 cells was measured in real-time, under basal conditions and in response to glucose, oligomycin and 2-DG for ECAR (panel a), and in response to mitochondrial drugs oligomycin, FCCP, Antimycin A and Rotenone for OCR (panel b). Indices of glycolytic pathway activation, calculated from PEA1 and PEA2 cells ECAR profile: basal ECAR, maximal ECAR and glycolytic capacity (see also Materials and Methods section). Indices of mitochondrial respiratory function, calculated from PEA1 and PEA2 cells OCR profile: basal OCR, ATP-linked OCR and maximal OCR (see also Materials and Methods section). For each profile is shown one representative out of three independent experiments. Data are expressed as mean \pm S.E.M. of three measurements, each of them in triplicates. (c and d) Kinetic profile of ECAR and OCR in PEA1 cells transfected with non-targeted control siRNA or TRAP1-directed siRNA was measured as described for PEA1 and PEA2 cells. For each profile, one representative out of three independent experiments is shown. Data are expressed as mean \pm S.E.M. of three measurements, each of them in triplicates. (Statistical analysis by two-tailed Mann-Whitney test)

our BEC index data, showing a shift toward OXPHOS in PEA1 cells upon TRAP1 silencing and in PEA2 chemoresistant cells, further supporting the previously described role of TRAP1 as a modulator of mitochondrial metabolism. ${ }^{20}$

On the basis of these observations, we aimed at verifying whether the metabolic features related to TRAP1 expression were accompanied by a change in expression or activity of key glycolytic enzymes, namely GAPDH and PFK. We then assessed the expression levels of these proteins by WB, comparing PEA1 and PEA2 cells and TRAP1-silenced PEA1 cells. GAPDH and PFK expression levels are decreased in PEA2-resistant cells compared with PEA1sensitive cells (Figure 4a), in keeping with impairment of glycolysis observed by Seahorse analysis in these cells. Conversely, TRAP1 silencing in PEA1 does not modify expression levels of such genes (Figure 4b), neither affects 
a

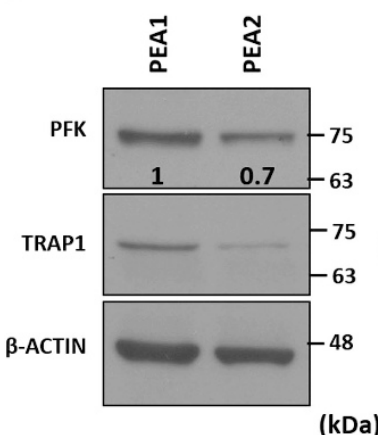

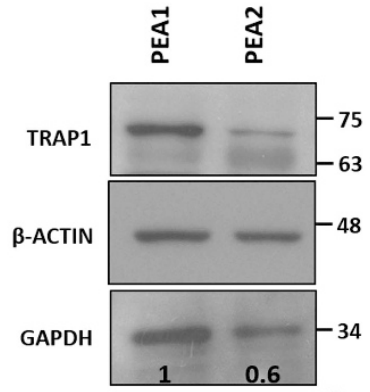

(kDa) b

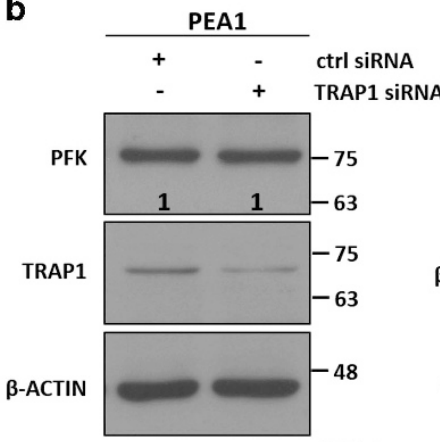

(kDa)

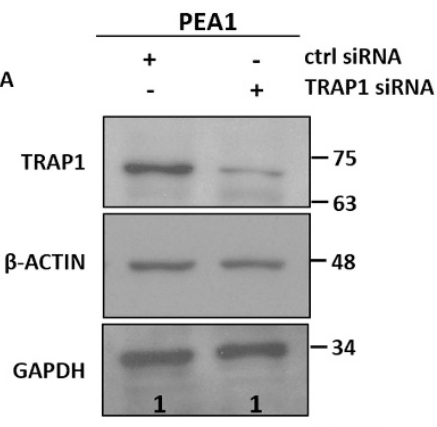

(kDa)
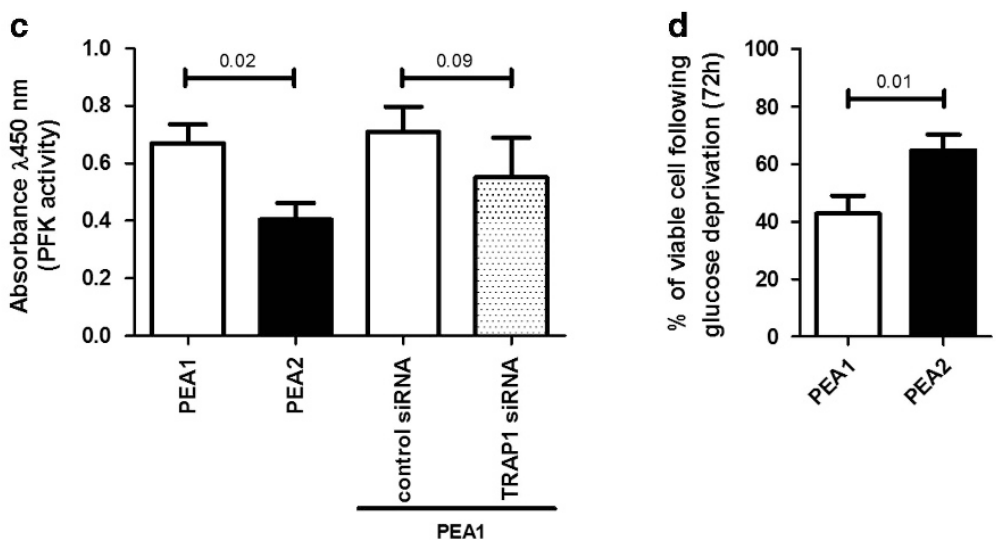

Figure 4 Glycolysis is decreased in resistant cells but not affected by TRAP1. (a and $\mathbf{b}$ ) Total lysates obtained from PEA1-sensitive and PEA2-resistant cells (a) or from PEA1 cells transfected for $72 \mathrm{~h}$ with non-targeted control siRNA or TRAP1-directed siRNA (b), were separated by SDS-PAGE and immunoblotted with the indicated antibodies. Numbers indicate densitometric band intensities, calculated by assuming protein levels of the control equal 1. Images are representative of three independent experiments. (c) PFK1 activity was measured by a colorimetric assay in PEA1-sensitive and PEA2-resistant cells and in PEA1 cells transfected for $72 \mathrm{~h}$ with non-targeted control siRNA or TRAP1-directed siRNA. Data are expressed as mean \pm S.E.M. from three independent experiments with technical triplicates each. Numbers above bars indicate the statistical significance ( $P$-value), based on the two-tailed Student's $t$-test. (d) Viability assays performed by MTT in PEA1 and PEA2 cells cultured in complete medium or in low-glucose medium for $72 \mathrm{~h}$. Absorbance was measured at $570 \mathrm{~nm}$ by subtracting background values at $690 \mathrm{~nm}$. Data are expressed as mean \pm S.E.M. from four independent experiments with technical triplicates each. Numbers above bars indicate the statistical significance ( $P$-value), based on the two-tailed Student's $t$-test

the activity of PFK, the rate-limiting enzyme of glycolysis, as demonstrated by colorimetric assays, whereas PFK activity is strongly decreased in PEA2 in comparison with PEA1 cells (Figure 4c). These data further confirm the metabolic profile obtained by Seahorse assays, showing that TRAP1 is directly involved in the regulation of mitochondrial respiration in these cells, but not in glycolysis.

The limited dependence of PEA2 cells from glucose sources is also confirmed by viability assays. PEA1 and PEA2 cells were grown for $72 \mathrm{~h}$ in a low-glucose medium, then cell viability was assessed by MTT assay. Results show that PEA2 cells are more resistant to glucose deprivation than their sensitive counterparts, thus demonstrating again their independence from glycolysis for survival (Figure 4d).

OXPHOS drives cisplatin resistance in low TRAP1 background. Once demonstrated the role of TRAP1 in OC metabolic shift, we tested the hypothesis that TRAP1 itself could be responsible for cisplatin sensitivity through this metabolic remodelling. Apoptosis was then investigated by caspase $3 / 7$ activity assays in TRAP1-silenced PEA1 cells upon cisplatin treatment: as shown in Figure 5a, TRAP1 silencing yields cells more resistant to cisplatin-induced caspase cleavage. PEA1 sh-TRAP1 stable transfectants are also more resistant to cisplatin treatment than controls (Supplementary Figure S1). Interestingly, glucose withdrawal induces cisplatin resistance in both control and TRAP1silenced PEA1 cells, thus suggesting that inhibition of glycolysis and metabolic shift toward OXPHOS may be responsible for the response to the drug (Figure 5a).

We then reasoned that, if TRAP1-silenced cells are more resistant to cisplatin and more addicted to OXPHOS, the inhibition of mitochondrial respiration may sensitize these cells to cisplatin-induced apoptosis. To test our hypothesis, we combined cisplatin treatment with subtoxic concentrations of metformin, a well-known antidiabetic drug, which is an inhibitor of complex I of the respiratory chain. ${ }^{26}$ Results show that metformin restores sensitivity to cisplatin in TRAP1-interfered PEA1 cells (Figure 5b). Accordingly, PEA2-resistant cells were resensitized to cisplatin treatment when coupled with metfor$\mathrm{min}$, as demonstrated by both viability (Figure $5 \mathrm{c}$ ) and caspase activity assays (Figure $5 \mathrm{~d}$ ). Interestingly, antineoplastic effects of metformin have been recently reported in $\mathrm{OC}$ models. ${ }^{27}$ These results were further confirmed by viability 
assays using, instead of metformin, the ATP synthase inhibitor oligomycin, which significantly potentiates the effect of Cisplatin (Supplementary Figure S2). Conversely, we asked whether cisplatin resistance could be directly induced by increased OXPHOS activity. To this aim, PEA1 cells were treated with the uncoupler agent FCCP, which increases
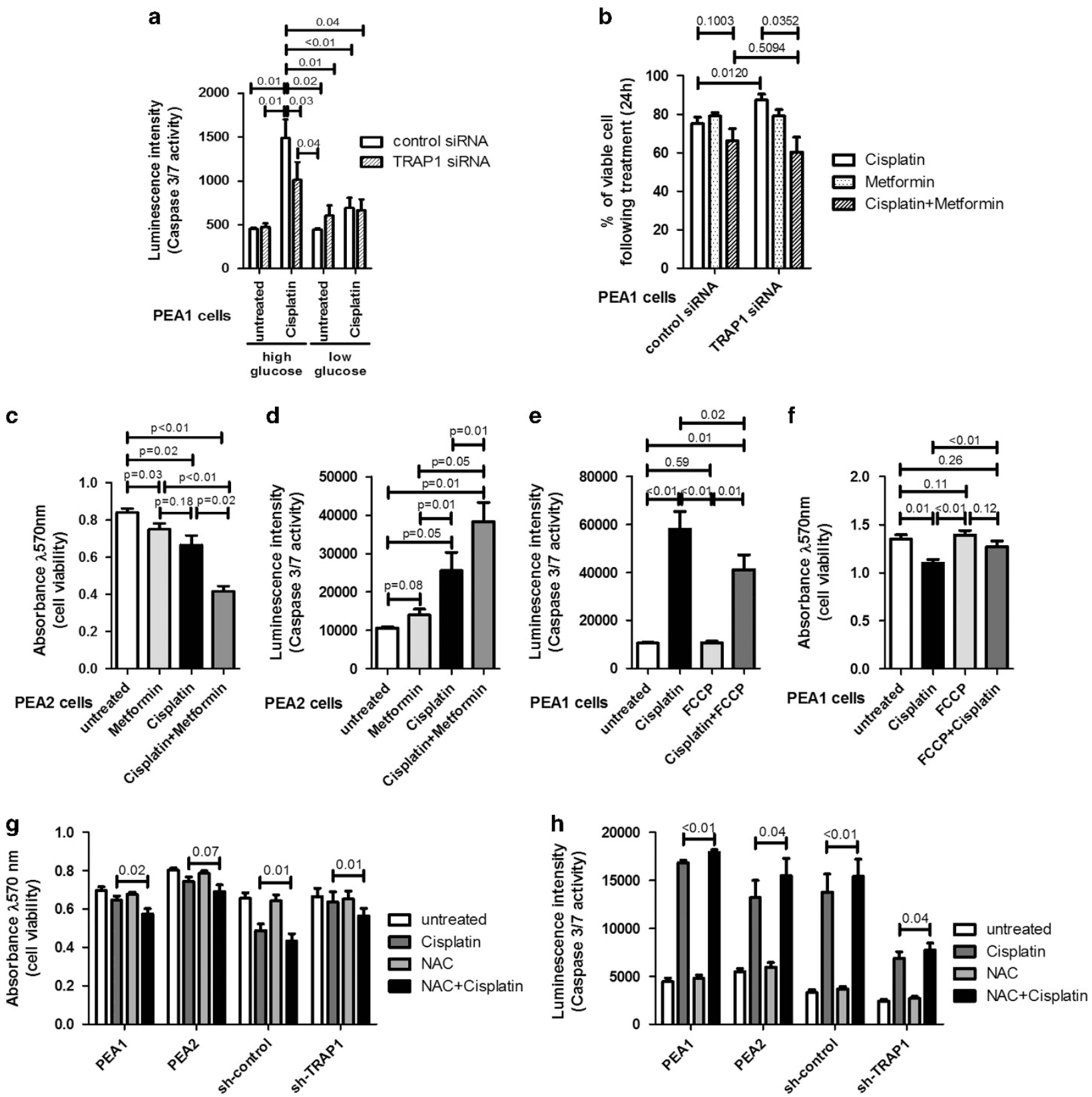

Figure 5 Cisplatin resistance in OC cells is mediated by oxidative metabolism. (a) PEA1 cells were transfected with non-targeted control siRNA or TRAP1-directed siRNA. Seventy-two hours after transfection, cells were placed in high-glucose $(4.5 \mathrm{~g} / \mathrm{l})$ or low-glucose $(1 \mathrm{~g} / \mathrm{l})$ medium and, after $1 \mathrm{~h}$, further treated for $24 \mathrm{~h}$ with $20 \mu \mathrm{M}$ cisplatin. Subsequently, apoptosis was measured by a luminescent caspase $3 / 7$ activity assay. Data are expressed as mean \pm S.E.M. from four independent experiments with technical triplicates each. (b) PEA1 cells were transfected with non-targeted control siRNA or TRAP1-directed siRNA. Seventy-two hours after transfection, cells were treated for $24 \mathrm{~h}$ with $20 \mu \mathrm{M}$ cisplatin, $10 \mathrm{mM}$ metformin or both. Subsequently, cell viability was measured by MTT assay. Data are expressed as mean \pm S.E.M. from three independent experiments with technical triplicates each. (c and d) PEA2 cells were treated with $40 \mu \mathrm{M}$ cisplatin, $10 \mathrm{mM}$ metformin or both for $24 \mathrm{~h}$, then cell viability was measured by MTT assay (c) and apoptosis was measured by a luminescent caspase $3 / 7$ activity assay (d). See also Supplementary Figure S1. Data are expressed as mean \pm S.E.M. from four independent experiments with technical triplicates each. (e and f) PEA1 cells were treated with $20 \mu \mathrm{M}$ cisplatin, $1.2 \mu \mathrm{M} \mathrm{FCCP}$ or both for $24 \mathrm{~h}$, then apoptosis was measured by caspase $3 / 7$ activity luminescent assay (e) and their viability was assessed by MTT assay (f). Data are expressed as mean \pm S.E.M. from five independent experiments with technical triplicates each. ( $\mathbf{g}$ and $\mathbf{~ h}$ ) Cells were pretreated for $1 \mathrm{~h}$ with NAC $1 \mathrm{mM}$, then treated with $20 \mu \mathrm{M}$ (PEA1) or $40 \mu \mathrm{M}$ cisplatin (PEA2) after NAC washout. After $24 \mathrm{~h}$, cell viability was measured by MTT assay ( $\mathbf{g}$ ) and apoptosis was measured by a luminescent caspase $3 / 7$ activity assay (h). Data are expressed as mean \pm S.E.M. from four (PEA1-PEA2) or six independent experiments (sh-control and sh-TRAP1 PEA1 cells) with technical triplicates each. In all panels, numbers above bars indicate the statistical significance ( $P$-value), based on the two-tailed Student's $t$-test 
respiration rate without affecting ATP cellular production, alone or in combination with cisplatin and, as expected, we demonstrated that FCCP reverts cisplatin apoptotic effect (Figures $5 \mathrm{e}$ and $\mathrm{f}$ ), thus supporting our idea of a metabolic involvement in cisplatin resistance of OC systems. Reactive oxygen species (ROS) production could reasonably lie between low TRAP1/high OXPHOS and cisplatin resistance. Indeed, ROS assays demonstrate that platinum-resistant PEA2 and TRAP1 knock-down PEA1 cells contain higher ROS levels than their respective counterparts (Supplementary Figure S3). Furthermore, both viability and apoptosis assays (Figures $5 \mathrm{~g}$ and $\mathrm{h}$ ) show that antioxidant tretament with $\mathrm{N}$-acetyl cysteine (NAC) sensitizes PEA1 and PEA2 cells, as well as TRAP1 knock-down PEA1 cells and control, to cisplatin-induced cell death. Altogether, these data reinforce the hypothesis that TRAP1 is involved in cisplatin sensitivity through metabolic remodelling in OC cells.

TRAP1-mediated metabolic shift elicits inflammatory response. As demonstrated by apoptosis and cell viability assays, inhibition of glycolysis by glucose deprivation (Figure 5a) and OXPHOS stimulation by FCCP treatment (Figures $5 \mathrm{e}$ and f) are per se able to induce cisplatin resistance in PEA1-sensitive cells. These results prompted us to search for the molecules directly responsible for the modulation of cisplatin function in these conditions. To this aim, we cultured PEA1 cells for $6 \mathrm{~h}$ in the absence of glucose, and then performed a whole-genome gene expression analysis as described in Materials and Methods section, by comparing glucose deprived to control cells. This approach led to the identification of a set of 2158 (1072 upregulated and 1086 downregulated) regulated genes (see Supplementary Table S1 for the full list of differentially regulated genes). The Ingenuity Pathway Analysis (IPA) software allowed us to identify, among the five most relevant regulated biofunctions, the aryl hydrocarbon receptor signalling and the glucocorticoid receptor signalling, two pathways involved in cellular immune response and inflammatory activation $^{28,29}$ (Figure 6a). Interestingly, previous literature reports that $\mathrm{OC}$ is characterized by an autocrine production of interleukins (ILs) and cytokines ${ }^{30}$ and recent studies associate metabolic reprogramming to IL signalling, ${ }^{31}$ which has been recently involved in cisplatin resistance. ${ }^{32}$ Starting from these observations, we selected the 11 most regulated genes in these two pathways according to Ingenuity software (Supplementary Table S2) for qPCR validation upon glucose deprivation, TRAP1 silencing and treatment with FCCP, an inducer of mitochondrial respiration (Figure 6b). Among the selected genes, qPCR analysis confirmed CSF2, HSPA1A, IL6 and SGK1 as significantly regulated upon a short-term $(6 \mathrm{~h})$ glucose deprivation; TRAP1 silencing yielded analogous regulation of $C S F 2$ and $I L 6$, and also resulted in significant regulation of IL8 and PLAU; similarly, FCCP treatment modulated the expression of CSF2, IL6 and PLAU in the same direction as glucose deprivation and TRAP1 silencing. Interestingly, regulation of CSF2 and IL6 was common to all treatments, thus suggesting a significant involvement of such genes in the observed phenomena. To further support these data, we analyzed the correlations between the genes validated in the gene expression analysis and TRAP1 levels by bioinformatic analysis of OC tissue database (see Supplementary Tables S3 and S4 for the full list of genes/proteins and their correlation coefficient with TRAP1). Remarkably, 3 of the 11 genes, namely IL6, PLAU, $M A P 2 K 2$, significantly correlated with TRAP1 in the OC RNAseq TCGA data set (Figure 6c): IL6 and PLAU increased upon TRAP1 silencing, according to our previous analysis (Figure 6b). Notably, PLAU and TRAP1 have also been reported to oppositely respond to anti-oestrogen-based therapy, with PLAU being downregulated by oestrogen, in contrast to the upregulation of TRAP1, ${ }^{33}$ which bears an oestrogen-responsive element in its promoter region. ${ }^{34}$ These findings strongly suggest that inhibition of glycolysis and increased oxidative metabolism in PEA1 cells stimulate the production of proinflammatory molecules, which may be responsible for cisplatin resistance.

Metabolism-driven inflammation induces platinum resistance. We hypothesized that the relevance of TRAP1-related signature in the onset of OC platinum resistance may be due to its peculiar ability to produce, in response to increased OXPHOS, proinflammatory cytokines, such as IL6 and IL8, which are usually secreted by immune cells. $^{35}$ To investigate this hypothesis, we performed a pathway analysis on the microarray profile of matched cell lines PEA1/PEA2, PEO1/PEO4/PEO6, PEO14/PEO23. ${ }^{36}$ Results showed that the inflammatory pathway and the activation of IL signalling are the most significant differentially regulated pathways between resistant/sensitive cell lines (Figure 7a).

As IL6, among all interleukins, resulted upregulated in all our tested conditions (glucose deprivation, FCCP treatment, TRAP1 silencing), and inversely correlated to TRAP1 in human OC samples, we tested whether treatment with human recombinant IL6 was sufficient to induce resistance to cisplatin in sensitive cells. To this aim, PEA1 cells were treated with $200 \mathrm{U} / \mathrm{ml}$ IL6 $4 \mathrm{~h}$ before cisplatin addition $(20 \mu \mathrm{M})$ for further $24 \mathrm{~h}$. Results showed that indeed PEA1 cells became more resistant to drug treatment, as demonstrated by reduced apoptosis sensitivity upon IL6 pretreatment (Figure 7b). To characterize at a molecular level the mechanisms/factors responsible for this phenomenon, we started from the recent whole-genome characterization of chemoresistant $\mathrm{OC},{ }^{37}$ showing that acquired resistance associated with overexpression of the multidrug resistance protein 1 (MDR1). Therefore, we focused on two members of this family: $A B C B 1 / M D R 1$, an ATP-dependent efflux transporter, whose involvement in tumour drug resistance has been widely reported, ${ }^{38}$ and $A B C B 2 / T A P 1$, which enhances pancreatic ductal adenocarcinoma drug resistance ${ }^{39}$ and, notably, resulted upregulated in all sets of PEA2/PEO4/PEO23 resistant cells in comparison with their matched sensitive counterparts. ${ }^{36}$ To this aim, we analyzed MDR1 and TAP1 levels by qPCR in PEA1 cells upon TRAP1 silencing and upon treatment with two different proinflammatory inducers, namely TNFa and IL6. As shown in Figure 7c, both TAP1 and MDR1 were upregulated by TNFa/IL6 exposure, and a significant induction of TAP1 is observed upon TRAP1 silencing, thus supporting the role of TRAP1/MDR1-TAP1 pathway in our study model. Accordingly, siRNA-mediated TAP1 silencing yielded an increased 
a
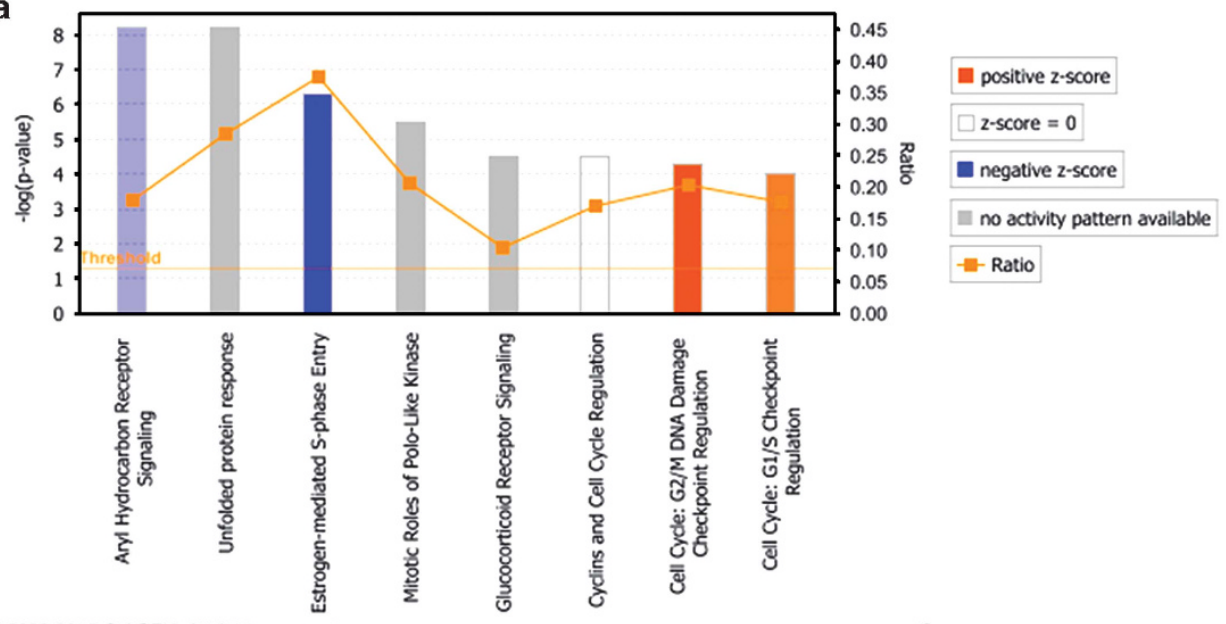

2000-2015 QIAGEN. All rights reserved.
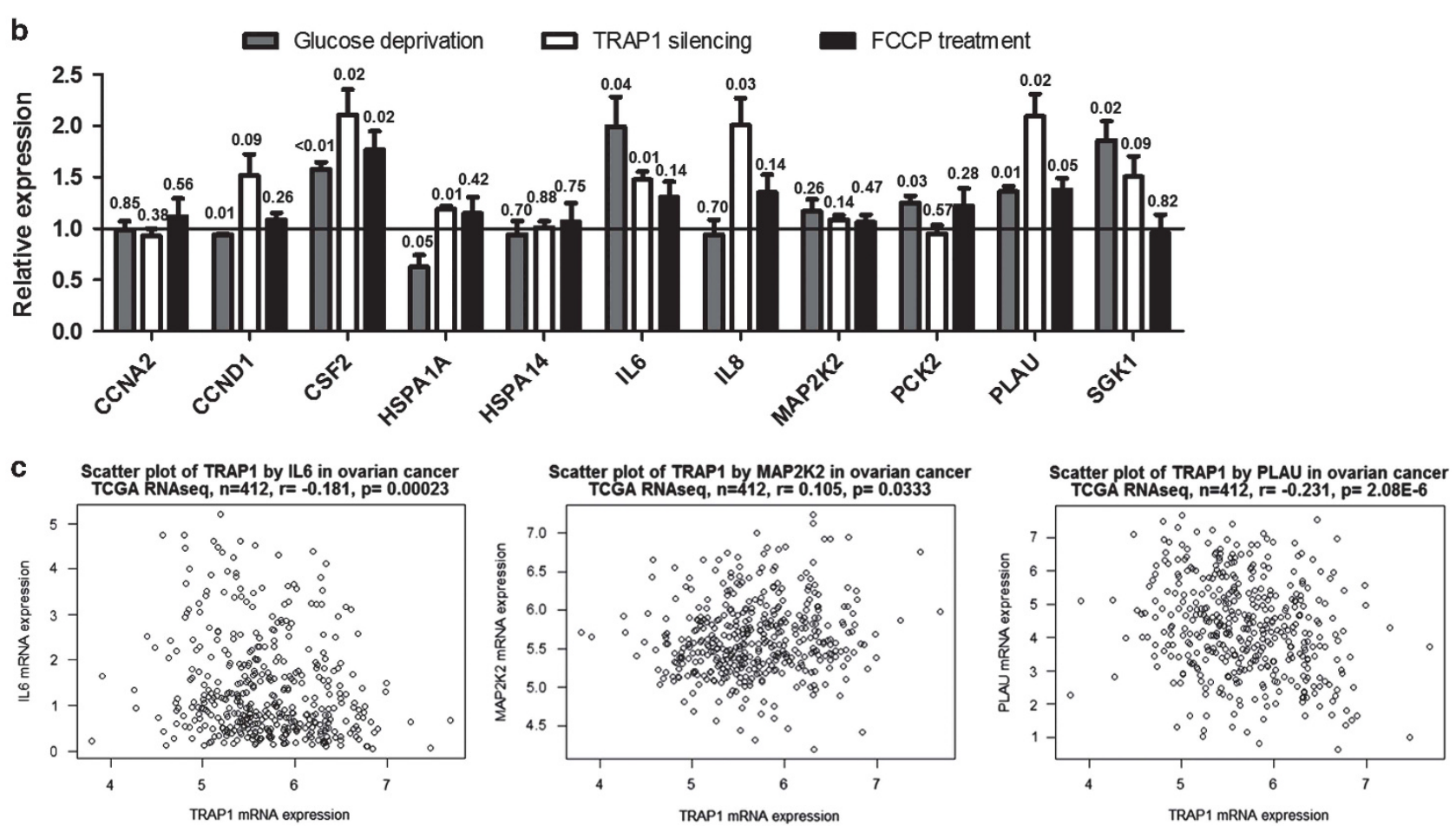

Figure 6 Metabolic remodelling of PEA1 cells elicits inflammatory response. (a) Pathway activity analysis (2000-2014 Ingenuity Systems, Inc.), representing the eight canonical pathways most differentially regulated in PEA1 cells upon Glucose deprivation (6h). Orange bars or blue bars predict an increase or decrease in a pathway's activity, respectively. Log $(P$-value) indicates statistical significance of selected pathway, z-score is used to infer the activation states of predicted pathways, and ratio indicates percentage of genes in analysis compared with the whole pathway gene list (see also Supplementary Table S1). (b) Real-time RT-PCR analysis of expression of 11 selected genes belonging to the glucocorticoid receptor signalling or the aryl hydrocarbon receptor signalling, following $6 \mathrm{~h}$ of glucose deprivation, upon siRNA-mediated TRAP1 silencing, and following $6 \mathrm{~h}$ of FCCP treatment in PEA1 cells (see also Supplementary Table S2). All data are expressed as mean \pm S.E.M. from four independent experiments with technical triplicates each. Numbers above bars indicate the statistical significance ( $P$-value), based on the one-sample $t$-test. Solid line indicate expression level of the relative control. (c) Correlation between TRAP1 expression and IL6, MAP2K2 and PLAU mRNA expression in TCGA data set. $P$-values determined by the Pearson's product moment correlation coefficient. See also Supplementary Table S3

sensitivity to cisplatin-induced apoptosis in PEA1 cells and slightly but significantly restored sensitivity to cisplatin in PEA2 cisplatin-resistant cells (Figure7d). Mechanistically, all these findings allowed us to identify ATP transporters as possible key proteins involved/responsible for platinum resistance in these OC cells.

\section{Discussion}

HGSOC is the most malignant form of $\mathrm{OC}$ and accounts for up to $70 \%$ of all OC cases. Currently, survival rates of patients with $\mathrm{OC}$ remain poor and $\mathrm{OC}$ continues to be a poorly understood disease. ${ }^{40}$ Indeed, OC experimental models are mostly unable to recapitulate the human disease, raising 
a

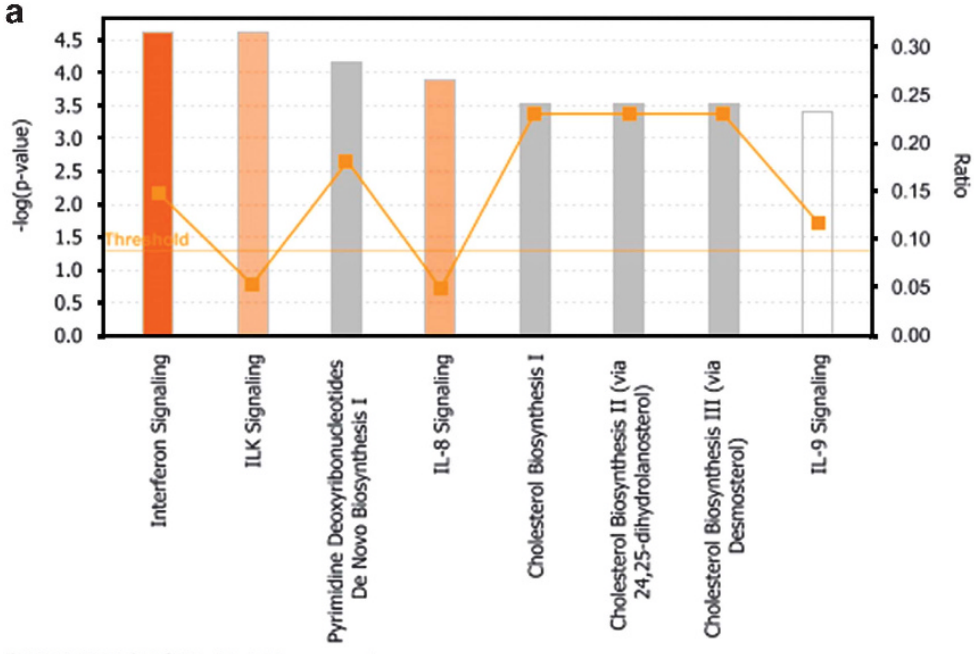

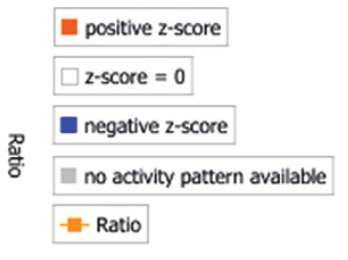

positive z-score

negative 2 -score

- $=$ Ratio

2000-2015 QIAGEN. All rights reserved.

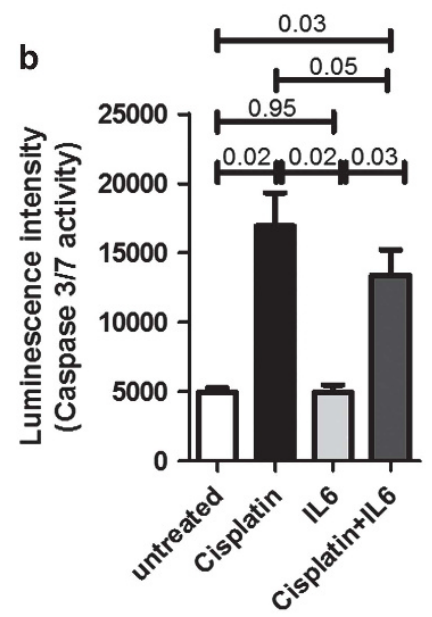

C

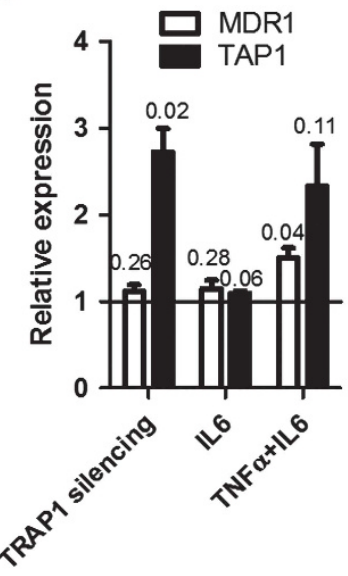

d

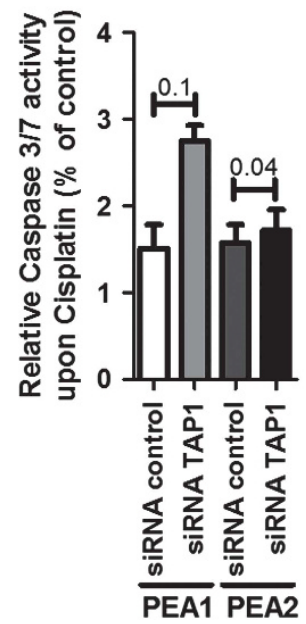

Figure 7 Activation of inflammatory pathways is responsible for cisplatin resistance. (a) Pathway activity analysis (2000-2014 Ingenuity Systems, Inc.), representing the eight canonical pathways most differentially regulated in PEA2/PEO4/PEO23 resistant cells in comparison with their matched sensitive counterparts. Orange bars or blue bars predict an increase or decrease in a pathway's activity, respectively. Log ( $P$-value) indicates statistical significance of selected pathway, $z$-score is used to infer the activation states of predicted pathways, and ratio indicates percentage of genes in analysis compared with the whole pathway gene list. (b) PEA1 cells were treated with $20 \mu \mathrm{M}$ cisplatin in the presence/absence of human IL6 $(200 \mathrm{U} / \mathrm{ml})$ for $24 \mathrm{~h}$, then apoptosis was measured by caspase $3 / 7$ activity luminescent assay. Data are expressed as mean \pm S.E.M. from four independent experiments with technical triplicates each. Numbers above bars indicate the statistical significance ( $P$-value), based on the two-tailed Student's $t$-test. (c) Real-time RT-PCR analysis of MDR1 and TAP1 expression in PEA1 cells $72 \mathrm{~h}$ after transfection with non-targeted control siRNA or TRAP1-directed siRNA or after 6-h treatment with human IL6 (200 U/ml) or both TNF $\alpha(20 \mathrm{ng} / \mathrm{ml})$ and IL6. Data are expressed as mean \pm S.E.M. from three independent experiments with technical triplicates each. Numbers above bars indicate the statistical significance ( $P$-value), based on one-sample $t$-test. Solid line indicate expression level of the relative control. (d) PEA1 and PEA2 cells were transfected with non-targeted control siRNA or TAP1-directed siRNA. Seventy-two hours, cells were treated for $24 \mathrm{~h}$ with $20 \mu \mathrm{M}$ cisplatin (PEA1) or $40 \mu \mathrm{M}$ cisplatin (PEA2). Subsequently, apoptosis was measured by a luminescent caspase $3 / 7$ activity assay. Data are expressed as mean \pm S.E.M. from three independent experiments with technical triplicates each. Numbers above bars indicate the statistical significance ( $P$-value), based on the two-tailed Student's $t$-test

debate about the need for better systems. ${ }^{41}$ Therefore, we started from a set of HGSOC tissue specimens and confirmed our data at molecular level taking advantage of multiple set of matched HGSOC cell lines (PEA1/PEA2, PEO1/PEO4/PEO6, PEO16/PEO23), derived from the same patient before (PEA1, PEO1, PEO16) and after (PEA2, PEO4, PEO6, PEO23) the development of platinum resistance. Moreover, we have repeatedly matched the obtained results with a large database of HGSOC biopsies by analyzing the correlation between expression data in RNA/protein arrays and metabolic and clinical data of patients, also further confirming all the findings in $47 \mathrm{HGSOC}$ tissue specimens. As a result, we found that lower levels of the molecular chaperone TRAP1 are associated with advanced disease and poor survival in HGSOC. This is surprising on the basis of the present literature, presenting TRAP1 as a component of the HSP90 mitochondrial survival pathway with antioxidant ${ }^{42}$ and antiapoptotic functions, ${ }^{43}$ and a very promising prospective target for novel cancer therapeutics. ${ }^{44}$ Recent advances in TRAP1 biology have shown that it functions as a network hub protein, involved 


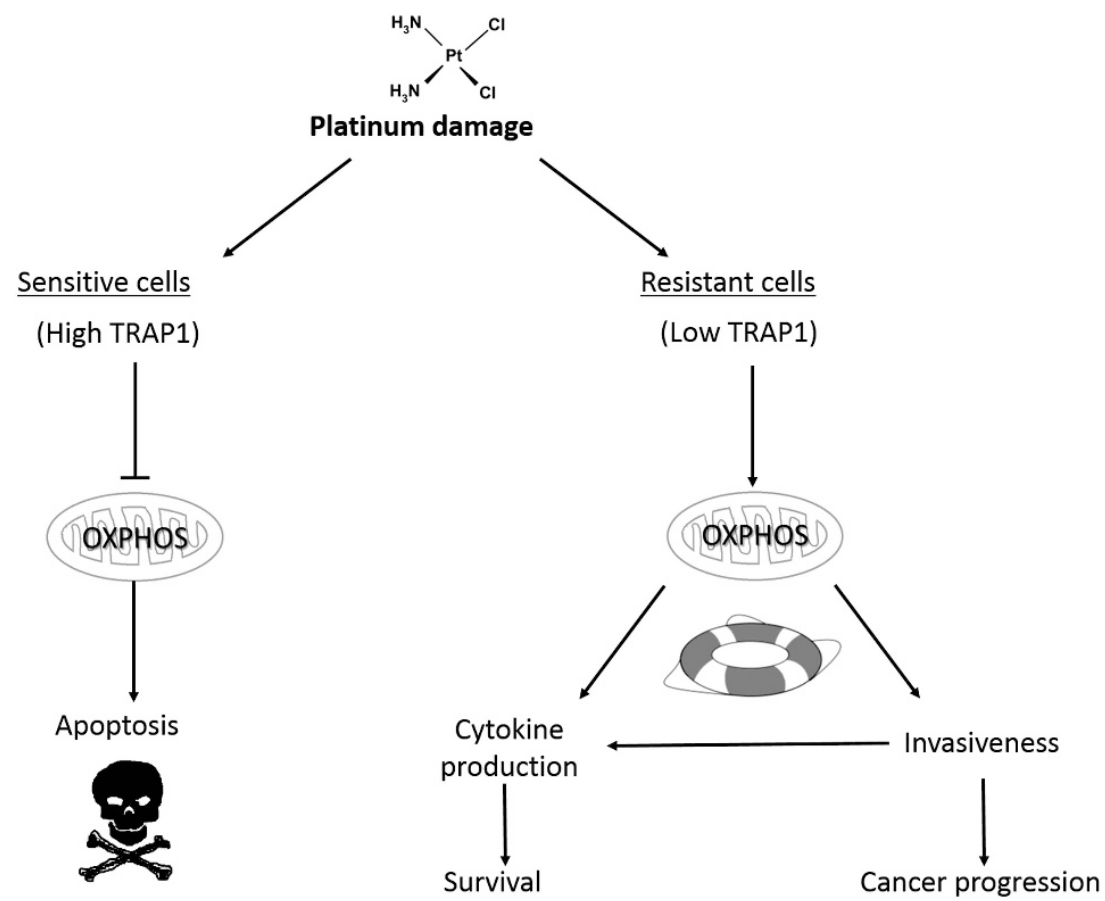

Figure 8 A model for the OXPHOS-mediated activation of survival pathways in low versus high TRAP1-expressing ovarian tumour cells following clinically acquired platinum resistance. Cells expressing low TRAP1 levels have increased OXPHOS activity, which is usually escaped by cancer cells in order to pursue the advantages of aerobic glycolysis. However, OC is characterized by an autocrine production of ILs and cytokines, mainly produced by immune cells, whose expression is stimulated by OXPHOS through an unknown mechanism. This elicits an inflammatory response, which is responsible for resistance to cisplatin

in most cellular processes responsible for the rewiring of tumour metabolism, including the regulation of protein synthesis and degradation, ${ }^{45,46}$ endoplasmic reticulum stress protection of cancer cells $s^{47,48}$ and the regulation of mitochondrial physiology and cancer cell metabolism. ${ }^{21}$ This apparent contradiction is unravelled by the observation that higher oxidative metabolism correlated with higher tumour grade in OC, and that cisplatin-resistant OC cells showed lower TRAP1 levels and increased OXPHOS and ROS levels. Accordingly, TRAP1 silencing in cisplatin-sensitive cells increased OCR/ROS, induced cisplatin resistance and, strikingly, this phenotype was reversible upon pharmacological inhibition of mitochondrial OXPHOS or ROS scavenging. For decades, decreased capacity for OXPHOS because of dysfunctional mitochondria and increased glycolysis have been considered as dominant in neoplastic cells; however, very recent observations challenge this view in a growing number of tumour systems, especially melanoma. ${ }^{49}$ Remarkably, recent data suggested that OC could be included in the list, showing that the most aggressive OC cell lines mostly depend on glutamine rather than on glucose ${ }^{5}$ and cancer stem cells from EOC patients privilege OXPHOS and resist glucose deprivation. ${ }^{6}$ Intriguingly, the first inverse correlations between TRAP1 and tumour stage was reported in bladder, clear cell renal cell and cervical carcinoma, ${ }^{18}$ with the latter mostly relying on OXPHOS for its energetic metabolism. ${ }^{19}$ In agreement, previous data correlated TRAP1 expression with favourable chemotherapy response and overall survival in a subset of ERa-positive OC patients. ${ }^{22}$
Our results show that the strict correlation between low TRAP1 expression and oxidative metabolism in OC cells is responsible for progression of disease and response to platinum-based therapy, through the activation of an inflammatory programme. Proinflammatory cytokines, such as IL6, are usually secreted by immune cells, but their expression has been documented in osteosarcomas, ${ }^{50} \mathrm{OC}$ cells $^{35}$ and head and neck squamous cell carcinoma cells. ${ }^{51}$ Some interesting observations show that elevated levels of IL6 in the sera and ascites of OC patients predict poor clinical outcome, ${ }^{52}$ IL8 and IL8 receptor have been proposed as potential prognostic biomarkers and therapeutic targets ${ }^{53}$ and have a role in platinum response in HGSOC. ${ }^{32}$ However, to our knowledge, no connection has ever been demonstrated between inflammation, metabolism and drug resistance. In this regard, a very recent work shows that loss of $\mathrm{p} 62$ (a NF- $\kappa \mathrm{B}$ regulator) in stromal fibroblasts of prostate cancer promoted tumour progression through a regulation of cellular redox, resulting in increased IL6 production. ${ }^{31} \mathrm{~A}$ model for the OXPHOS-mediated activation of survival pathways in the establishment of platinum resistance in $\mathrm{OC}$ is shown in Figure 8. Mechanistically, we identified MDR subfamily members (TAP1 and MDR1) as key mediators of such metabolism-driven, inflammation-induced drug resistance. Consistently, according to a recent whole-genome characterization of chemoresistant $\mathrm{OC},{ }^{37}$ acquired resistance associated with overexpression of the drug efflux pump MDR1. It has been proposed that platinum-resistant HGSOC clones may arise from low-abundance subset of cells within the sensitive tumour through a selection process, rather than 
through adaptation. ${ }^{54}$ Metabolic characterization of similar sub-populations of tumorigenic and multidrug resistant cells in other cancer types revealed their dependency on mitochondrial respiration, ${ }^{49}$ which is consistent with our data. In this regard, the antidiabetic metformin, is already under investigation for possible applications in oncology ${ }^{55}$ and has been recently reported as an effective antineoplastic agent in OC models. ${ }^{27}$ In conclusion, we suggest that metabolic features of tumour cells in the peculiar environment of $\mathrm{OC}$ can predict cisplatin sensitivity and that TRAP1, bioenergetic index and proinflammatory molecules can serve as predictive/ prognostic biomarkers of therapeutic outcome. In this context, we propose the targeting of OXPHOS to improve cisplatin efficacy in tumours with increased oxidative metabolism.

\begin{abstract}
Materials and Methods
Cell cultures. The paired HGSOC cell lines PEA1 and PEA2 have been described elsewhere. ${ }^{24}$ Cell line verification was by Identifiler kit (Applied BiosystemsThermo Fisher Scientific, Waltham, MA, USA). TRAP1 stable interfered cells were obtained by transfecting PEA1 cells with TRAP1 (5'-TGCTGTTGACAGTGAGC GACCCGGTCCCTGTACTCAGAAATAGTGAAGCCACAGATGTATTTCTGAGTACAGG GACCGGGCTGCCTACTGCCTCGGA-3') or scrambled (sequence containing no homology to known mammalian genes) short hairpin RNAs (Open Biosystem, Dharmacon, Arese, Italy) and isolating positive clones by selection with Puromycin (Sigma-Aldrich, Milano, Italy) $1 \mu \mathrm{g} / \mathrm{ml}$. All lines were maintained in RPMl-1640 media with $10 \%$ foetal bovine serum, penicillin, streptomycin, glutamine at $37^{\circ} \mathrm{C} / 5 \% \mathrm{CO}_{2}$. Cell lines are routinely monitored in our laboratory by microscopic morphology check.
\end{abstract}

Transfection procedures. TRAP1 transient silencing was performed with siRNAs purchased from Qiagen (cat. no. SI00115150); sense strand: 5'-CGGUCC CUGUACUCAGAAATT-3'; antisense strand: 5'-UUUCUGAGUACAGGGACCGG G-3'. For control experiments, cells were transfected with a similar amount of nontargetin control siRNA (Qiagen, Milano, Italy; cat. no. SI03650318). Transient transfections of siRNAs were performed using HiPerFect Transfection Reagent (Qiagen) according to the manufacturer's protocol.

Tumour lysates. Tumour samples were manually cut in ice cold T-PER Lysis Buffer (Thermo Scientific, Waltham, MA, USA) and sonicated. Samples were centrifuged twice at $10000 \times g$ for $10 \mathrm{~min}$ at $4{ }^{\circ} \mathrm{C}$ and supernatants were collected and quantified.

Western blot analysis. Equal amounts of protein from cell lysates and tumour specimens were subjected to SDS-PAGE and transferred to a PVDF membrane (Merck-Millipore, Darmstadt, Germany). Where indicated, protein levels were quantified by densitometric analysis using the software ImageJ. ${ }^{56}$ The following antibodies were used: anti-TRAP1 (sc-13557), anti- $\beta$-actin (sc-69879), anti-GAPDH (sc-69778), anti-ATP5B (sc-16690), anti-HSP60 (sc-1052), from Santa Cruz Biotechnology (Dallas, TX, USA); anti-PFK1 (NBP2-32170) from Novus Bio (Abingdon, UK).

RNA extraction and real-time RT-PCR. Total RNA extraction procedures were performed by using TRI Reagent (Sigma-Aldrich, product code T9424), following the manufacturer's instruction. The following primers were used for PCR analysis. TRAP1: forward 5'-GACGCACCGCTCAACAT-3', reverse 5'-CACATCAAA CATGGACGGTTT-3'; ACTIN: forward 5'-CCTCACCCTGAAGTACCCCA-3', reverse 5'-TCGTCCCAGTTGGTGACGAT-3'; IL8: forward 5'-CCAGTCTTGTCATTGCCAGC -3', reverse 5'-TGACTGTGGAGTTTTGGCTGT-3'; IL6: forward 5'-TGCAAT;AAC CACCCCTGACC-3', reverse 5'-CAATCTGAGGTGCCCATGCT-3'; CSF2: forward 5'-GTCATCTTGGAGGGACCAAGG-3', reverse 5'-CCATGCCTGTATCAGGGTCA G-3'; HSPA1A: forward 5'-GGGCCTTTCCAAGATTGCTG-3', reverse 5'-TGCAAAC ACAGGAAATTGAGAACT-3'; CCND1: forward 5'-TGCTGCGAAGTGGAAACCAT C-3', reverse 5'-CACTTCTGTTCCTCGCAGAC-3'; PLAU: forward 5'-CTGTGTGG ACTGTGATGCCA-3', reverse 5'-CAGTGCTGGTCACAGGTCAT-3'; CCNA2: forward 5'-ACACAGTCACGGGACAAAGC-3', reverse 5'-TGCTGTGGTGCTTTGAGGTA-3'; SGK1: forward 5'-CGTTAGAGTGCCGCCTTAGA-3', reverse 5'-TTCGTCATCACA GCCCAGAC-3'; MAP2K2: forward 5'-CGGACCTGAAGATGCTCACA-3', reverse
5'-TTCAGCCGCAGGGTTTTACA-3'; HSPA14: forward 5'-GGGTGTGGACGAATCA GGAG-3', reverse 5'-ATATGCTTCCAGGGGCTTGC-3'; PCK2: forward 5'-CCCCAA GGACTTCTGGGAAC-3', reverse 5'-TTGGGCAGATCCTGGTTGAC-3'; MDR1: forward 5'-TCCTCAGTCAAGTTCAGAGTCTTCA-3', reverse 5'-TCTCCACTTGATG ATGTCTCTCACT-3'; TAP1: forward 5'-CCTCCTTTCCAAGCTCCTCG-3', reverse 5'-GGCCAGCATATGCCTTCAGT-3'. When possible, primers were designed to be intron spanning. The reaction conditions were $95^{\circ} \mathrm{C}$ for 5 min followed by 45 cycles of $15 \mathrm{~s}$ at $95^{\circ} \mathrm{C}$ and $1 \mathrm{~min}$ at $60^{\circ} \mathrm{C}$. Actin was chosen as the internal control. In PCR analyses performed upon TRAP1 silencing, RNAs were collected $72 \mathrm{~h}$ after siRNA transfection; in PCR analyses performed upon FCCP treatment and glucose deprivation, RNAs were collected after 6-h treatment.

Metabolic analyses. The BEC indices of the cell lines and tissue samples were calculated, based on digital density scanning of western blots as described above, by dividing the ratio of $\beta$ F1ATPase to HSP60 with the GAPDH value.

The metabolic profile has been evaluated in PEA1, PEA2 cell lines and in PEA1 cells silenced for TRAP1 (72 h after transfection). Real-time measurements of OCR and ECAR were made using an XF-96 Extracellular Flux Analyzer (Seahorse Bioscience, North Billerica, MA, USA). Cells were plated in XF-96 plates (Seahorse Bioscience) at the concentration of $4 \times 10^{4}$ cells per well and cultured for $12 \mathrm{~h}$ in DMEM medium supplemented with $5 \%$ FBS. OCR was measured in XF media (non-buffered DMEM medium, containing $10 \mathrm{mM}$ glucose, $2 \mathrm{mM}$ L-glutamine and $1 \mathrm{mM}$ sodium pyruvate), under basal conditions and in response to $2.5 \mu \mathrm{M}$ oligomycin, $1.5 \mu \mathrm{M}$ of carbonylcyanide-4-(trifluoromethoxy)-phenylhydrazone (FCCP) and $1 \mu \mathrm{M}$ of Antimycin and Rotenone (all from Sigma-Aldrich). Indices of mitochondrial respiratory function were calculated from OCR profile: basal OCR (before addition of oligomycin), ATP-linked OCR (calculated as the difference between basal OCR rate and oligomycin-induced OCR rate) and maximal OCR (calculated as the difference of FCCP rate and antimycin+rotenone rate). ECAR was measured in XF media in basal condition and in response to $10 \mathrm{mM}$ glucose, $2.5 \mu \mathrm{M}$ oligomycin and $100 \mathrm{mM}$ of 2-DG (all from Sigma-Aldrich). Indices of glycolytic pathway activation were calculated from ECAR profile: basal ECAR (after the addition of glucose), maximal ECAR (after the addition of oligomycin) and glycolytic capacity (calculated as the difference of oligomycin-induced ECAR and 2-DG-induced ECAR). Experiments with the Seahorse system have been performed with the following assay conditions: 3-min mixture; 3-min wait; and 3-min measurement; metabolic parameters were then calculated. Data are expressed as mean \pm S.E.M.

PFK activity was assessed by using Phosphofructokinase Activity Colorimetric Assay Kit (Sigma, product code MAK093), following the manufacturer's instructions. When assessing PFK activity upon TRAP1 silencing, the assay was performed $72 \mathrm{~h}$ after siRNA transfection.

Detection of intracellular ROS in live cells was performed by using the Fluorometric Intracellular Ros Kit (MAK145, Sigma), according to the manufacturer's instructions.

Cell treatments and apoptosis and viability assays. In glucose deprivation experiments, cells were plated in monolayer in complete medium. After seeding, medium was replaced with a low-glucose (1 g/l) RPMI medium (Sigma). In cell viability and apoptosis assays, PEA1 cells were treated with $20 \mu \mathrm{M}$ cisplatin for $24 \mathrm{~h}$, whereas PEA2 cells were treated with $40 \mu \mathrm{M}$ cisplatin for $24 \mathrm{~h}$. In sensitization experiments, cells were treated for $24 \mathrm{~h}$ with metformin $10 \mathrm{mM}$, or contemporary with metformin and cisplatin for $24 \mathrm{~h}$, with the same concentrations indicated above. In acquired resistance experiments, cell were pretreated with FCCP $1.2 \mu \mathrm{M}$ or with human IL6 $200 \mathrm{U} / \mathrm{ml}$ for $4 \mathrm{~h}$, before adding cisplatin for further $24 \mathrm{~h}$.

Cell viability was measured by MTT assay by using the In vitro toxicology assay kit (Sigma, product code TOX1-1KT), following the manufacturer's instructions. Apoptosis was measured using the Caspase-Glo 3/7 assay (Promega, Milano, Italy, product code G8090) and was performed according to the manufacturer's instructions.

Gene expression analysis. Gene expression analysis (sample preparation, hybridization, staining, scanning and data preprocessing/quantile normalization) were performed as described in Condelli et al. ${ }^{12}$ Differently expressed genes (DEGs) between cells cultured in low- and high-glucose medium for $6 \mathrm{~h}$ were selected with differential score (DiffScore) cutoff set at $\pm 13(P<0.05)$ and genes with log fold change $\leq-0.3$ or $\geq 0.3$ were further analyzed. Microarray data were submitted to Array Express under accession number E-MTAB-3799. The DEGs list (composed by 909 genes, 462 upregulated and 447 downregulated) was used to evaluate the functional behaviour in terms of Biological Processes and Canonical Pathway performing an enrichment analysis with IPA. 
Furthermore, we confirmed all the results, by previous gene expression data ${ }^{36}$ of multiple sensitive/resistant matched cell lines (PEA1/PEA2, PE01/PEO4/PE06, PE016/PEO23) derived from the same patient before and after the development of platinum resistance. Data were extracted from Supplementary Table and Canonical Pathway of differentially expressed genes were evaluated with IPA.

Statistics. Serous OC cases from Tothill data set (Affymetrix, Santa Clara, CA, USA U133 plus2.0 microarray, GSE9891) and TCGA data set (level 3 data, total RNA sequencing and Reverse Phase Protein Array) were used to correlate TRAP1 mRNA expression with stage, grade and PFS in Figures 1a-c, and to correlate with the expression of other genes in Figure 6c. Wilcoxon rank sum test was used to compare gene expression between two groups. Kruskal-Wallis test with 10000 permutation was used to compare gene expression among more than two groups. Log-rank test was used to determine the significance of the survival analysis. Pearson's product moment correlation coefficient was used to correlate the mRNA expression of two genes.

The paired Student's t-test was used to establish the statistical significance of differences between gene expression levels in qPCR and between viability/apoptosis/ PFK activity levels under TRAP1 silencing or drug treatments. Two-tailed MannWhitney test was used to determine statistical significance of metabolic measurements reported in Figure 3.

Patients and study approval. Patients' samples were collected under the Imperial College London Tissue Bank project number R15024 (TRAP1 regulates bioenergetics features, cisplatin resistance and epithelial-mesenchymal transition in $\mathrm{OC}$ ) in accordance with the Imperial College London guidelines. Express written informed consent to use biological specimens for investigational procedures was obtained from all patients. All experimental protocols were approved by Hammersmith and Queen Charlotte's and Chelsea Research Ethics Committee.

\section{Conflict of Interest}

The authors declare no conflict of interest.

Acknowledgements. This work was supported by the Associazione Italiana per la Ricerca sul Cancro (AIRC) (grant IG2015-16738 to ML and FE), by the Italian Ministry of Health (grant GR-2010-2310057) to FM, by POR Campania FSE and FSR 2007-2013, Projects CRËME and STRAIN, LR 5/2002 annuity 2008 to FE. GM is supported by grants from the Fondazione Italiana Sclerosi Multipla (FISM) no. 2012/ $\mathrm{R} / 11$, the European Union IDEAS Programme European Research Council Starting Grant "menTORingTregs" n. 310496 and ASI (Italian Space Agency) no. 2014-033-R.O.

\section{Author contributions}

DSM and MRA designed research, conducted experiments, analyzed data and wrote the manuscript; DA, DF and CP conducted experiments and analyzed data; IA, RA, FM and CM conducted experiments; VS and HL acquired and analyzed data; $E Z$ and CR provided sample's lysates and analyzed data; HG, G Marone, ES and G Matarese critically revised the paper; ML and FE designed research, analyzed data and wrote the manuscript.

1. Haq R, Shoag J, Andreu-Perez P, Yokoyama S, Edelman H, Rowe GC et al. Oncogenic BRAF regulates oxidative metabolism via PGC1 $\alpha$ and MITF. Cancer Cell 2013; 23: 302-315.

2. Vazquez F, Lim JH, Chim H, Bhalla K, Girnun G, Pierce K et al. PGC1 $\alpha$ expression defines a subset of human melanoma tumors with increased mitochondrial capacity and resistance to oxidative stress. Cancer Cell 2013; 23: 287-301.

3. LeBleu VS, O'Connell JT, Gonzalez Herrera KN, Wikman H, Pantel K, Haigis MC et al. PGC-1alpha mediates mitochondrial biogenesis and oxidative phosphorylation in cancer cells to promote metastasis. Nat Cell Biol 2014; 16: 992-1003.

4. Tan AS, Baty JW, Dong LF, Bezawork-Geleta A, Endaya B, Goodwin J et al. Mitochondrial genome acquisition restores respiratory function and tumorigenic potential of cancer cells without mitochondrial DNA. Cell Metab 2015; 21: 81-94.

5. Yang L, Moss T, Mangala LS, Marini J, Zhao H, Wahlig S et al. Metabolic shifts toward glutamine regulate tumor growth, invasion and bioenergetics in ovarian cancer. Mol Syst Biol 2014; 10: 728.

6. Pastò A, Bellio C, Pilotto G, Ciminale V, Silic-Benussi M, Guzzo G et al. Cancer stem cells from epithelial ovarian cancer patients privilege oxidative phosphorylation, and resist glucose deprivation. Oncotarget 2014; 5: 4305-4319.

7. Siegel R, Ma J, Zou Z, Jemal A. Cancer statistics, 2014. CA Cancer J Clin 2014; 64: 364.
8. Costantino E, Maddalena F, Calise S, Piscazzi A, Tirino V, Fersini A et al. TRAP1, a nove mitochondrial chaperone responsible for multi-drug resistance and protection from apoptotis in human colorectal carcinoma cells. Cancer Lett 2009; 279: 39-46.

9. Megger DA, Bracht T, Kohl M, Ahrens M, Naboulsi W, Weber F et al. Proteomic differences between hepatocellular carcinoma and nontumorous liver tissue investigated by a combined gel-based and label-free quantitative proteomics study. Mol Cell Proteomics 2013; 12 : 2006-2020.

10. Agorreta J, Hu J, Liu D, Delia D, Turley H, Ferguson DJ et al. TRAP1 regulates proliferation, mitochondrial function, and has prognostic significance in NSCLC. Mol Cancer Res 2014; 12 660-669.

11. Leav I, Plescia J, Goel HL, Li J, Jiang Z, Cohen RJ et al. Cytoprotective mitochondrial chaperone TRAP-1 as a novel molecular target in localized and metastatic prostate cancer. Am J Pathol 2010; 176: 393-401.

12. Condelli V, Piscazzi A, Sisinni L, Matassa DS, Maddalena F, Lettini G et al. TRAP1 is involved in BRAF regulation and downstream attenuation of ERK phosphorylation and cell-cycle progression: a novel target for BRAF-mutated colorectal tumors. Cancer Res 2014; 74: 6693-6704.

13. Condelli V, Maddalena F, Sisinni L, Lettini G, Matassa DS, Piscazzi A et al. Targeting TRAP1 as a downstream effector of BRAF cytoprotective pathway: a novel strategy for human BRAF-driven colorectal carcinoma. Oncotarget 2015; 6: 22298-22309.

14. Maddalena F, Sisinni L, Lettini G, Condelli V, Matassa DS, Piscazzi A et al. Resistance to paclitxel in breast carcinoma cells requires a quality control of mitochondrial antiapoptotic proteins by TRAP1. Mol Oncol 2013; 7: 895-906.

15. Sisinni L, Maddalena F, Lettini G, Condelli V, Matassa DS, Esposito F et al. TRAP1 role in endoplasmic reticulum stress protection favors resistance to anthracyclins in breast carcinoma cells. Int J Oncol 2014; 44: 573-582.

16. Caino MC, Chae YC, Vaira V, Ferrero S, Nosotti M, Martin NM et al. Metabolic stress regulates cytoskeletal dynamics and metastasis of cancer cells. J Clin Invest 2013; 123. 2907-2920.

17. Agliarulo I, Matassa DS, Amoroso MR, Maddalena F, Sisinni L, Sepe L et al. TRAP1 controls cell migration of cancer cells in metabolic stress conditions: correlations with AKT/p70S6K pathways. Biochim Biophys Acta 2015; 1853: 2570-2579.

18. Yoshida S, Tsutsumi S, Muhlebach G, Sourbier C, Lee MJ, Lee S et al. Molecular chaperone TRAP1 regulates a metabolic switch between mitochondrial respiration and aerobic glycolysis. Proc Natl Acad Sci USA 2013; 110: 1604-1612.

19. Moreno-Sánchez R, Rodríguez-Enríquez S, Marín-Hernández A, Saavedra E. Energy metabolism in tumor cells. FEBS J 2007; 274: 1393-1418.

20. Rasola A, Neckers L, Picard D. Mitochondrial oxidative phosphorylation TRAP(1)ped in tumor cells. Trends Cell Biol 2014; 24: 455-463.

21. Sciacovelli M, Guzzo G, Morello V, Frezza C, Zheng L, Nannini N et al. The mitochondria chaperone TRAP1 promotes neoplastic growth by inhibiting succinate dehydrogenase. Cell Metab 2013; 17: 988-999.

22. Aust S, Bachmayr-Heyda A, Pateisky P, Tong D, Darb-Esfahani S, Denkert C et al. Role of TRAP1 and estrogen receptor alpha in patients with ovarian cancer -a study of the OVCAD consortium. Mol Cancer 2012; 11: 69.

23. Matassa DS, Amoroso MR, Maddalena F, Landriscina M, Esposito F. New insights into TRAP1 pathway. Am J Cancer Res 2012; 2: 235-248.

24. Langdon SP, Lawrie SS, Hay FG, Hawkes MM, McDonald A, Hayward IP et al. Characterization and properties of nine human ovarian adenocarcinoma cell lines. Cancer Res 1988; 48: 6166-6172.

25. Hjerpe E, Egyhazi Brage S, Carlson J, Frostvik Stolt M, Schedvins K, Johansson $\mathrm{H}$ et al. Metabolic markers GAPDH, PKM2, ATP5B and BEC-index in advanced serous ovarian cancer. BMC Clin Pathol 2013; 13: 30.

26. Owen MR, Doran E, Halestrap AP. Evidence that metformin exerts its anti-diabetic effects through inhibition of complex 1 of the mitochondrial respiratory chain. Biochem J 2000; 348 (Pt 3): 607-614.

27. Lengyel E, Litchfield LM, Mitra AK, Nieman KM, Mukherjee A, Zhang Y et al. Metformin inhibits ovarian cancer growth and increases sensitivity to paclitaxel in mouse models. Am J Obstet Gynecol 2015; 212: 479.e10.

28. Vogel CF, Khan EM, Leung PS, Gershwin ME, Chang WL, Wu D et al. Cross-talk between aryl hydrocarbon receptor and the inflammatory response: a role for nuclear factor- $\kappa \mathrm{B}$. J Biol Chem 2014; 289: 1866-1875.

29. Coutinho AE, Chapman KE. The anti-inflammatory and immunosuppressive effects of glucocorticoids, recent developments and mechanistic insights. Mol Cell Endocrinol 2011; 335: 2-13.

30. Son DS, Kabir SM, Dong YL, Lee E, Adunyah SE. Inhibitory effect of tumor suppressor p53 on proinflammatory chemokine expression in ovarian cancer cells by reducing proteasomal degradation of $\mathrm{I}_{\kappa} \mathrm{B}$. PLOS One 2012; 7: e51116.

31. Valencia T, Kim JY, Abu-Baker S, Moscat-Pardos J, Ahn CS, Reina-Campos M et al. Metabolic reprogramming of stromal fibroblasts through p62-mTORC1 signaling promotes inflammation and tumorigenesis. Cancer Cell 2014; 26: 121-135.

32. Stronach EA, Cunnea P, Turner C, Guney T, Aiyappa R, Senthuran Jeyapalan S et al. The role of Interleukin-8 (IL-8) and IL-8 receptors in platinum response in high grade serous ovarian carcinoma. Oncotarget 2015; 6: 31593-31603.

33. Walker G, MacLeod K, Williams AR, Cameron DA, Smyth JF, Langdon SP. Estrogenregulated gene expression predicts response to endocrine therapy in patients with ovarian cancer. Gynecol Oncol 2007; 106: 461-468. 
34. O'Donnell AJ, Macleod KG, Burns DJ, Smyth JF, Langdon SP. Estrogen receptor-alpha mediates gene expression changes and growth response in ovarian cancer cells exposed to estrogen. Endocr Relat Cancer 2005; 12: 851-866.

35. Wang TH, Chan YH, Chen CW, Kung WH, Lee YS, Wang ST et al. Paclitaxel (Taxol) upregulates expression of functional interleukin- 6 in human ovarian cancer cells through multiple signaling pathways. Oncogene 2006; 25: 4857-4866.

36. Stronach EA, Alfraidi A, Rama N, Datler C, Studd JB, Agarwal R et al. HDAC4-regulated STAT1 activation mediates platinum resistance in ovarian cancer. Cancer Res 2011; 71: 4412-4422

37. Patch AM, Christie EL, Etemadmoghadam D, Garsed DW, George J, Fereday S et al. Wholegenome characterization of chemoresistant ovarian cancer. Nature 2015; 521: 489-494.

38. Binkhathlan Z, Lavasanifar A. P-glycoprotein inhibition as a therapeutic approach for overcoming multidrug resistance in cancer: current status and future perspectives. Curr Cancer Drug Targets 2013; 13: 326-346.

39. Xu M, Li L, Liu Z, Jiao Z, Xu P, Kong X et al. ABCB2 (TAP1) as the downstream target of SHH signaling enhances pancreatic ductal adenocarcinoma drug resistance. Cancer Lett 2013; 333: $152-158$.

40. Vaughan S, Coward JI, Bast RC Jr, Berchuck A, Berek JS, Brenton JD et al. Rethinking ovarian cancer: recommendations for improving outcomes. Nat Rev Cancer 2011; 11: 719-725.

41. Lengyel E, Burdette JE, Kenny HA, Matei D, Pilrose J, Haluska P et al. Epithelial ovarian cancer experimental models. Oncogene 2014; 33: 3619-3633.

42. Montesano Gesualdi N, Chirico G, Pirozzi G, Costantino E, Landriscina M, Esposito F. Tumor necrosis factor-associated protein 1 (TRAP-1) protects cells from oxidative stress and apoptosis. Stress 2007; 10: 342-350.

43. Landriscina M, Laudiero G, Maddalena F, Amoroso MR, Piscazzi A, Cozzolino F et al. Mitochondrial chaperone Trap1 and the calcium binding protein Sorcin interact and protect cells against apoptosis induced by antiblastic agents. Cancer Res 2010; 70: 6577-6586.

44. Amoroso MR, Matassa DS, Sisinni L, Lettini G, Landriscina M, Esposito F. TRAP1 revisited: novel localizations and functions of a 'next-generation' biomarker (review). Int J Oncol 2014; 45: 969-977.

45. Matassa DS, Amoroso MR, Agliarulo I, Maddalena F, Sisinni L, Paladino S et al. Translational control in the stress adaptive response of cancer cells: a novel role for the heat shock protein TRAP1. Cell Death Dis 2013; 4: e851.

46. Matassa DS, Agliarulo I, Amoroso MR, Maddalena F, Sepe L, Ferrari MC et al. TRAP1dependent regulation of p70S6K is involved in the attenuation of protein synthesis and cell migration: relevance in human colorectal tumors. Mol Oncol 2014; 8: 1482-1494.

47. Amoroso MR, Matassa DS, Laudiero G, Egorova AV, Polishchuk RS, Maddalena F et al. TRAP1 and the proteasome regulatory particle TBP7/Rpt3 interact in the endoplasmic reticulum and control cellular ubiquitination of specific mitochondrial proteins. Cell Death Differ 2012; 19: 592-604.
48. Matassa DS, Arzeni D, Landriscina M, Esposito F. ER stress protection in cancer cells: the multifaceted role of the heat shock protein TRAP1. Endoplasmic Reticulum Stress Dis 2014; 1 ISSN (Online) 2300-4266.

49. Viale A, Corti D, Draetta GF. Tumors and mitochondrial respiration: a neglected connection. Cancer Res 2015; 75: 3685-3686.

50. Duan Z, Lamendola DE, Penson RT, Kronish KM, Seiden MV. Overexpression of IL-6 but not IL-8 increases paclitaxel resistance of U-2OS human osteosarcoma cells. Cytokine 2002; 17: 234-242.

51. Poth KJ, Guminski AD, Thomas GP, Leo PJ, Jabbar IA, Saunders NA. Cisplatin treatment induces a transient increase in tumorigenic potential associated with high Interleukin-6 expression in head and neck squamous cell carcinoma. Mol Cancer Ther 2010; 9: 2430-2439.

52. Cohen S, Bruchim I, Graiver D, Evron Z, Oron-Karni V, Pasmanik-Chor M et al. Platinumresistance in ovarian cancer cells is mediated by IL-6 secretion via the increased expression of its target clAP-2. J Mol Med (Berl) 2013; 91: 357-368.

53. Browne A, Sriraksa R, Guney T, Rama N, Van Noorden S, Curry E et al. Differential expression of IL-8 and IL-8 receptors in benign, borderline and malignant ovarian epithelial tumours. Cytokine 2013; 64: 413-421.

54. Cooke SL, Ng CKY, Melnyk N, Garcia MJ, Hardcastle T, Temple J et al. Genomic analysis of genetic heterogeneity and evolution in high-grade serous ovarian carcinoma. Oncogene 2010; 29: 4905-4913.

55. Pollak MN. Investigating metformin for cancer prevention and treatment: the end of the beginning. Cancer Discov 2012; 2: 778-790.

56. Schneider CA, Rasband WS, Eliceiri KW. NIH Image to ImageJ: 25 years of image analysis. Nat Methods 2012; 9: 671-675.

This work is licensed under a Creative Commons Attribution-NonCommercial-NoDerivs 4.0 International License. The images or other third party material in this article are included in the article's Creative Commons license, unless indicated otherwise in the credit line; if the material is not included under the Creative Commons license, users will need to obtain permission from the license holder to reproduce the material. To view a copy of this license, visit http://creativecommons.org/licenses/by-nc-nd/4.0/

(C) The Author(s) 2016

Supplementary Information accompanies this paper on Cell Death and Differentiation website (http://www.nature.com/cdd) 